Gynäkologe 2016 · 49:498

DOI 10.1007/s00129-016-3910-5

Online publiziert: 20. Juni 2016

๑) Springer-Verlag Berlin Heidelberg 2016

CrossMark

\author{
W. Jonat ${ }^{1} \cdot$ K. Diedrich ${ }^{2,3}$ \\ 'Universitätsklinikum Schleswig Holstein Campus Kiel, Kiel, Deutschland \\ 2 amedes experts, Facharzt-Zentrum für Kinderwunsch, pränatale Medizin, Hamburg, Deutschland \\ ${ }^{3}$ Universitätsfrauenklinik Lübeck, Lübeck, Deutschland
}

\title{
Aktuelle gesundheitspolitische Entwicklung in der Frauenheilkunde
}

Die Frauenheilkunde und Geburtshilfe nimmt in der akademischen, wie auch in der klinischen, allgemeinen Medizin eine herausragende Rolle ein. Geprägt durch den Anspruch "der Hausarzt der Frau zu sein", war und ist es unser aller Ziel, als Frauenärzte durch exzellente Forschung und Umsetzung der Ergebnisse in den klinischen Alltag eine optimale Betreuung unserer Patientinnen zu gewährleisten.

Die Breite unseres Fachs, die sich im Säulenmodell wiederspiegelt und vom Beginn des Lebens bis zu seinem Ende reicht, macht immer wieder eine umfangreiche ethische Diskussion über die Sinnhaftigkeit unsere Tuns notwendig.

Diese häufig nicht nur kontrovers, sondern naturgemäß auch hoch emotional geführten Debatten sind sicher nicht einfach, zeigen aber auch, wie sehr wir alle bemüht sind, den richtigen Weg auch bei schwierigen grundsätzlichen Fragen des Lebens zu finden und gemeinsam mit unseren Patientinnen zu gehen.

Die heute geführten Diskussionen zum Erhalt unseres Faches und zur Definition der richtigen Strukturen sind bedingt durch die Notwendigkeit, auf die Veränderung unseres Faches sachgerecht zu reagieren. Diese Auseinandersetzungen haben unser Fach immer wieder geprägt.
Wie gehen wir im Spannungsfeld sektorenübergreifender Behandlungen miteinander um? Welches ist die beste Krebsvorsorge? Oder: Brauchen wir an Universitäten reproduktionsmedizinische Einheiten? Ist die Urogynäkologie eine eigenständige Säule unseres Faches?

Für all diese Fragen gibt es keine einfachen Antworten. Es bedarf auf unseren Tagungen immer wieder der Notwendigkeit, hierüber kollegial zu diskutieren und auch unter Eingehen schwieriger Kompromisse gemeinsame Positionen zu finden.

Neben diesen teils internen und durchaus auch hausgemachten Problemen ist unser Fach natürlich - ebenso wie die gesamte Medizin - immer wieder abhängig vom politischen Umfeld und den Plänen der jeweiligen politischen Mehrheiten.

Im vorliegenden Heft beschäftigten sich die Autoren mit einigen dieser Problemfelder. Besonders für unser Fachgebiet Reproduktionsmedizin zeigen sich hier unterschiedliche Einschätzungen der Protagonisten.

Wir bedanken uns bei allen beteiligten Autoren, die bereit waren, an diesem schwierigen Thema mitzuarbeiten. Unser Ziel ist es, mit diesem Heft Gedankenanstöße zu geben, nicht Lösungen anzubieten.
Weiterführende Diskussion und Wiederaufnahme der Themen sind in $\mathrm{Zu}$ kunft notwendig. Nur so wird unser Fach seine herausragende Bedeutung in akademischen und klinischen Medizin behalten.

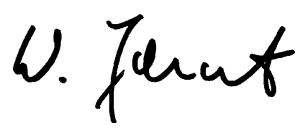

Walter Jonat

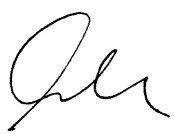

Klaus Diedrich

\section{Korrespondenzadresse}

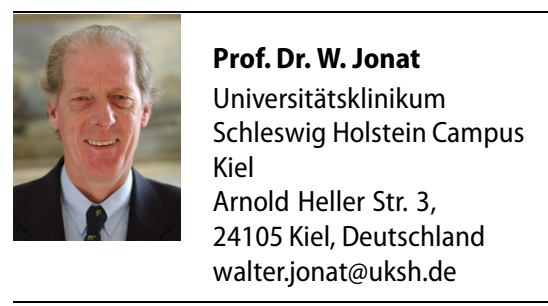

Interessenkonflikt. W. Jonat und K. Diedrich geben an, dass kein Interessenkonflikt besteht. 